\title{
Forced Convective of Micropolar Fluid on a Stretching Surface of Another Quiescent Fluid
}

\author{
${ }^{1}$ N. A. Majid, ${ }^{2}$ N. F. Mohammad*, ${ }^{3}$ A. R. M. Kasim and ${ }^{4}$ S. Shafie \\ ${ }^{1,2}$ Department of Computational and Theoretical Sciences, International Islamic University Malaysia \\ 25200 Kuantan, Pahang, Malaysia \\ ${ }^{3}$ Faculty of Industrial Sciences \& Technology, Universiti Malaysia Pahang \\ 26300 Gambang, Kuantan, Pahang, Malaysia \\ ${ }^{4}$ Department of Mathematical Sciences, Faculty of Science, Universiti Teknologi Malaysia \\ 81310 Skudai, Johor, Malaysia \\ *Corresponding author: farahain@iium.edu.my
}

Article history

Received: 28 September 2019

Received in revised form: 8 July 2019

Accepted: 16 September 2019

Published on line: 1 December 2019

\begin{abstract}
In this paper, the problem of forced convection flow of micropolar fluid of lighter density impinging orthogonally on another heavier density of micropolar fluid on a stretching surface is investigated. The boundary layer governing equations are transformed from partial differential equations into a system of nonlinear ordinary differential equations using similarity transformation and solved numerically using dsolve function in Maple software version 2016. The velocity, microrotation and temperature of micropolar fluid are analyzed. It is found that both upper fluid and lower fluid display opposite behaviour when micropolar parameter $K$ various with strong concentration $n=0, \operatorname{Pr}=7$ and stretching parameter $\lambda=0.5$. The results also show that stretching surface exert the force that increasing the velocity of micropolar fluid.
\end{abstract}

Keywords Micropolar fluid; stretching surface; quiescent fluid; forced convection.

Mathematics Subject Classification 76B99, 76R05.

\section{Introduction}

Theory of micropolar fluid was initiated by Eringen in year 1965 extending the famous equation of viscous fluid which is Navier-Stokes equation. Micropolar fluid is a non-Newtonian fluid that contained microstructure which experienced translational motion and rotational motion. Micropolar fluid theory also able to explain some fluid phenomena that contain suspended fluid additives such as animal blood, liquid crystal, paint, body fluid, colloidal fluid, magnetic fluids, cloud with dust, muddy fluids and polymer that cannot be explained by Navier-Stokes equation. Due to the wide applications, many researchers concentrate on micropolar as subject of study such as Rees and Bassom [1], Ahmadi [2], Chawla [3], Guram and Smith [4], Peddieson [5], Takhar and Soundalgekar [6], Ishak et al. [7], Ariffin et al. [8] and Shelukhin and Neverov [9]. 
Details about micropolar fluid can be found on Ariman et al. [10], Ariman et al. [11], Willson [12], Lukaszewicz [13] and Eremeyev et al. [14].

The problem of heat transfer in boundary layer flow is an active research field considering its many applications such as transpiration cooling, drag reduction, bearing and radial diffusers, thermal recovery of oil, the design of thrust bearings and radial diffusers, material drying, laser pulse heating and more [15]. In case of micropolar fluid, Eringen [16] extended problem of micropolar fluid with addition of thermal effect when he published The Theory of Thermofluidic. Gorla et al. [17] analyzed the heat transfer in micropolar boundary layer flow over a flat plate for both constant wall temperature and constant heat flux. Balaram and Sastri [18] discussed about free convection of micropolar fluid flow. On the other hand, a mathematical model of forced convection in micropolar fluid flow over a wavy surface was proposed by Cheng and Wang [19].

The boundary layer flow of shrinking surfaces is very useful in polymer extrusion, drawing of copper wires, continuous stretching of plastic films, hot rolling, glass fiber and many more especially in engineering field. Sakiadis [20] introduced the problem of moving surface. Miklavcic and Wang [22] initiated the investigation on shrinking sheet where they found that the solution of shrinking sheet is possible to exist if the stagnation point is considered or there is exist adequate suction. However, those studies are works only for Newtonian fluid. For micropolar fluid case, Soundalgekar and Takhar [23] analyzed flow of micropolar fluid past a continuously moving plate. In addition, Chiam [24] studied the micropolar fluid flow over a stretching sheet. While, $\mathrm{Na}$ and Pop [25] worked on boundary-layer flow of a micropolar fluid due to a stretching wall. The topic of stagnation-point flow over a shrinking sheet in a micropolar fluid was attempted by Ishak et al. [26].

The research continue to develop for convective flow and heat transfer over a stretching or shrinking sheet when Crane [21] analyzed the flow on stretching sheet. The application of this area can be seen in industrial and engineering process such as extrusion, glass fiber, glass blowing, paper production, extraction of polymer and rubber sheets. Besides, the rate of heat transfer at the stretching surface also give impact on the quality of final product. Yacob and Ishak [27] extended this work to micropolar fluid over a shrinking sheet. They found that stronger suction is required in order to a solution exist for micropolar fluid compared to Newtonian fluid.

Previous studies have primarily concentrated on boundary layer flow of one fluid model only. For two fluids model, the stagnation flow of surface of a quiescent fluid was investigated by Wang [28]. In different study, Reza and Gupta [29] studied MHD stagnation-point of an electrically conducting fluid on the surface of another quiescent fluid. A few years later, Rohni et al. [30] performed numerical analysis of stagnation-point of a fluid on a shrinking surface of another quiescent fluid. Recently, Reza et al. [31] presented their work on stagnation point flow and heat transfer for a viscoelastic fluid impinging on a quiescent fluid. Later, Isa and Mohammad [32] discover the relationship of dusty fluid on a stretching sheet of another quiescent fluid. But, none of the works mentioned involving two micropolar fluid. From our knowledge, the topic of forced convection of micropolar fluid over stretching surface of another quiescent fluid is still has not been investigated yet. 


\section{Problem Formulation}

Consider an incompressible micropolar fluid of density $\rho_{1}$, dynamic viscosity $\mu_{1}$, vortex viscosity $\mu_{1}$, spin-gradient viscosity $\gamma_{1}$ and microinertia density $j_{1}$ impinging orthogonally on a stretching surface of another quiescent, heavier incompressible micropolar fluid of density $\rho_{2}$, dynamic viscosity $\mu_{2}$, vortex viscosity $\kappa_{2}$, spin-gradient viscosity $\gamma_{2}$ and microinertia density $j_{2}$. $T_{w}$ and $T_{\infty}$ are temperature of surface and free stream temperature respectively. A sketch of the physical problem is shown in Figure 1. Let $\left(x, y_{1}\right)$ denote the Cartesian coordinates for the upper fluid with $x=0$ as the symmetry plane, and $x$-axis is taken along the interface between the two fluids. It is assumed that the surface is stretched or shrinked with the velocity $u_{w}(x)=c x$, where $c$ is a constant that $c>0$ indicates a stretching sheet. The coordinate system for the lower fluid is $\left(x, y_{2}\right)$ as shown in Figure 1 . Note that the $z$-axis is normal to the $\left(x, y_{1}\right)$ plane.

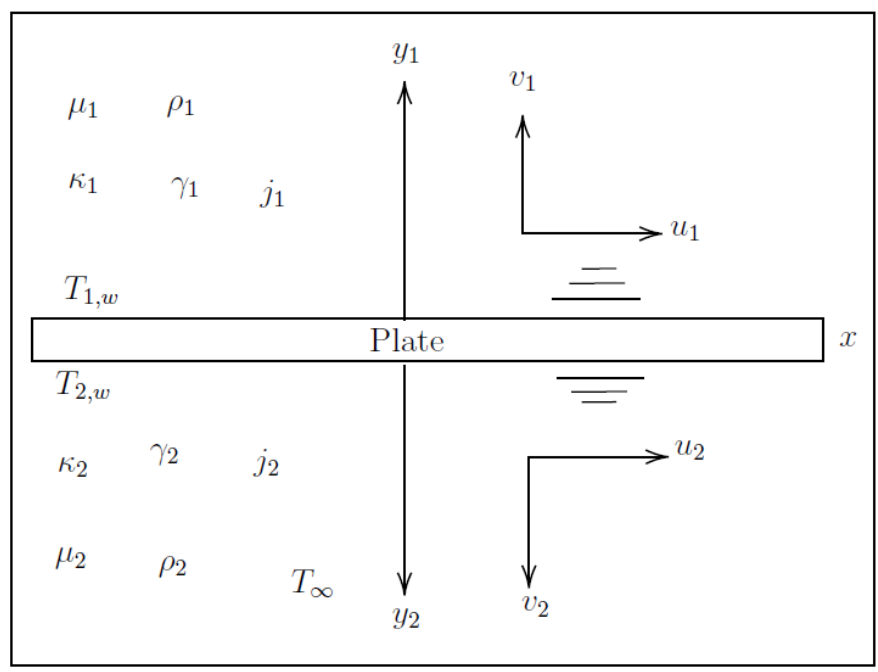

Figure 1: Physical Coordinate

Under the boundary layer approximations, the governing equations of continuity, momentum, angular momentum and energy are,

$$
\begin{gathered}
\frac{\partial u_{i}}{\partial x}+\frac{\partial v_{i}}{\partial y_{i}}=0 \\
u_{i} \frac{\partial u_{i}}{\partial x}+v_{i} \frac{\partial u_{i}}{\partial y_{i}}=U_{i} \frac{d U_{i}}{d x}+\frac{\mu_{i}+\kappa_{i}}{\rho_{i}} \frac{\partial^{2} u_{i}}{\partial y_{i}^{2}}+\frac{\kappa_{i}}{\rho_{i}} \frac{\partial N_{i}}{\partial y_{i}} \\
\rho_{i} j_{i}\left(u_{i} \frac{\partial N_{i}}{\partial x}+v_{i} \frac{\partial N_{i}}{\partial y_{i}}\right)=\gamma_{i} \frac{\partial^{2} N_{i}}{\partial y_{i}^{2}}-\kappa_{i}\left(2 N_{i}+\frac{\partial u_{i}}{\partial y_{i}}\right), \\
u_{i} \frac{\partial T_{i}}{\partial x}+v_{i} \frac{\partial T_{i}}{\partial y_{i}}=\frac{k_{0}}{\rho_{i} c_{p}} \frac{\partial^{2} T_{i}}{\partial y_{i}^{2}}
\end{gathered}
$$

subject to boundary conditions

$$
\begin{aligned}
& u_{i}(x)=c x, v_{i}=0, N_{i}=-n \frac{\partial u_{i}}{\partial y_{i}}, T_{i}=T_{i, w} \text { at } y_{i}=0 \\
& u_{1}(x) \rightarrow a x, u_{2}(x) \rightarrow 0, N_{i} \rightarrow 0, T_{i} \rightarrow T_{\infty} \text { as } y_{i} \rightarrow \infty
\end{aligned}
$$


where $u_{i}$ and $v_{i}$ are the velocity along $x$ and $y_{i}$ axes, $U_{i}=a x$ is free stream velocity, $\mu_{i}$ is vortex viscocity, $\kappa_{i}$ is vortex viscosity, $N_{i}$ is the component of microrotation vector normal to $x-y$ plane, $\rho_{i}$ is density of micropolar fluid, $j_{i}$ is microinertia density, $T_{i}$ is the fluid temperature, $\gamma_{i}$ is spin gradient viscocity, $k_{0}$ is thermal conductivity, $c_{p}$ is specific heat and $n$ is a constant such that $0 \leq n \leq 1$. From Jena and Mathur [33], the strong concentration case $(n=0)$ represents the concentrated particle flows in which the microelements close to the wall surface are unable to rotate. According to Ahmadi [2], the weak concentration case $(n=1 / 2)$ indicates the vanishing of the anti-symmetrical part of the stress tensor. The case $n=1$, as suggested by [5], is used for the modeling of turbulent boundary layer flows. In this paper, we consider the cases of $n=0$ (strong concentration) and $n=1 / 2$ (weak concentration). Further, we assume that $\gamma_{i}$ is defined as

$$
\gamma_{i}=\left(u_{i}+\kappa_{i} / 2\right) j_{i}=\mu_{i}\left(1+K_{i} / 2\right) j_{i}
$$

where $i=1,2$ is refering to upper fluid and lower fluid. Furthermore, $K_{i}$ is micropolar parameter, defined by

$$
K_{i}=\frac{\kappa_{i}}{\mu_{i}}
$$

For the upper fluid, we take

$$
\begin{aligned}
& u_{1}=a x f^{\prime}(\eta), v_{1}=-\sqrt{a \nu_{1}} f(\eta), \\
& N_{1}=a x \sqrt{a / \nu_{1}} g(\eta), \eta=y_{1} / \sqrt{\nu_{1} / a}, \theta_{1}=\frac{T_{1}-T_{\infty}}{T_{1, w}-T_{\infty}}
\end{aligned}
$$

and for lower fluid

$$
\begin{aligned}
& u_{2}=a x F^{\prime}(\xi), v_{2}=-\sqrt{a \nu_{2}} F(\xi), \\
& N_{2}=a x \sqrt{a / \nu_{2}} G(\xi), \xi=y_{2} / \sqrt{\nu_{2} / a}, \theta_{2}=\frac{T_{2}-T_{\infty}}{T_{2, w}-T_{\infty}},
\end{aligned}
$$

where $a$ is a constant, $\eta$ and $\xi$ are similarity variables and a prime denotes differentiation with coresspond to $\eta$ and $\xi$ respectively . Meanwhile, $\nu_{1}$ and $\nu_{2}$ is kinematic viscocity for upper fluid and lower fluid. Clearly with $u_{1}$ and $v_{1}$ given in (8), the equation of continuity (1) is satisfied. Similarly, for the lower fluid, with $u_{2}$ and $v_{2}$ given as in (9), it is readily seen that the continuity equation (1) is identically satisfied. Using (8), equations (2) - (4) are transformed into ordinary differential equation for the upper fluid flow

$$
\begin{gathered}
\left(1+K_{1}\right) f^{\prime \prime \prime}+f f^{\prime \prime}-f^{\prime 2}+K_{1} g^{\prime}+1=0, \\
\left(1+K_{1} / 2\right) g^{\prime \prime}+f g^{\prime}-f^{\prime} g-K_{1}\left(2 g+f^{\prime \prime}\right)=0, \\
\theta_{1}^{\prime \prime}+\operatorname{Pr} f \theta_{1}^{\prime}=0,
\end{gathered}
$$

with the boundary conditions

$$
f^{\prime}(\eta) \rightarrow 1, g(\eta) \rightarrow 0, \theta_{1}(\eta) \rightarrow 0 \text { as } \eta \rightarrow \infty
$$

Similarly, using (9), equations (2) - (4) for the lower fluid, we obtain

$$
\left(1+K_{2}\right) F^{\prime \prime \prime}+F F^{\prime \prime}-F^{2}+K_{2} G^{\prime}=0,
$$




$$
\begin{gathered}
\left(1+K_{2} / 2\right) G^{\prime \prime}+F G^{\prime}-F^{\prime} G-K_{2}\left(2 G+F^{\prime \prime}\right)=0, \\
\theta_{2}^{\prime \prime}+\operatorname{Pr} F \theta_{2}^{\prime}=0,
\end{gathered}
$$

with the boundary conditions

$$
F^{\prime}(\xi) \rightarrow 0, G(\xi) \rightarrow 0, \theta_{2}(\xi) \rightarrow 0 \text { as } \xi \rightarrow \infty
$$

It is also worth to mention that when $K_{1}=K_{2}=0$ the governing equation is reduced to Newtonian fluid [34]. At the interface, $\eta=\xi=0$, and so the continuity of velocity at the interface gives

$$
\begin{aligned}
& f(0)=0, f^{\prime}(0)=\lambda, F(0)=0, F^{\prime}(0)=\lambda, \\
& g(0)=-n f^{\prime \prime}(0), \theta_{1}(0)=1, \theta_{2}(0)=1, G(0)=-n F^{\prime \prime}(0),
\end{aligned}
$$

where $\lambda=c / a>0$ is the constant stretching parameter. It should be mentioned that equations (10) - (12) and (14) - (16) are subject to the boundary conditions (13), (17) and (18) have no closed form analytical solutions.

\section{Numerical Procedure}

The system of governing equations (10) - (12) and (14) - (16) was solved numerically. We are using built-in dsolve function that executed in Maple software version 2016. This built-in function uses finite difference method with Richardson's extrapolation as default method for solving boundary-value problems (BVP). The implemented algorithm is shown as in figure 2. Finite difference method is a method that used to solve boundary-value problems. Meanwhile Runge-Kutta-Fehlberg is one of the methods that used to solve initialvalue problems. Therefore, finite difference method is employed since this mathematical model involving boundary-value problem. On the other hand, Richardson's extrapolation is a technique that often applied with other method to improve the accuracy of a solution. Therefore, when Richardson's extrapolation is used with finite difference method, the accuracy of approximation to the solution of boundary-value problem can be improved.

\section{Results and Discussion}

From the numerical results obtained, we investigated the velocity, microrotation and the temperature of micropolar fluid in strong concentration $(n=0)$ and weak concentration $(n=0.5)$ where the micropolar parameter for both upper and lower fluid are the same $\left(K=K_{1}=K_{2}\right)$. According to Lok et al. [35], the flow over shrinking surface $(\lambda<0)$ is not restricted within a boundary layer. For that reason, we only consider for stretching surface $(\lambda>0)$. Table 1 represents the comparison values of skin friction when stretching parameter $\lambda$ varies and $K=0$ with Rosali et al. [36] for validation purpose. It is shown that the current numerical outcomes agree with the previous work.

Figures 3 - 5 and table 2 show velocity, skin friction, microrotation and temperature for both upper fluid and lower fluid respectively when micropolar parameter $K$ varies with concentration $n=0$, stretching parameter $\lambda=0.5$ and $\operatorname{Pr}=7$ which represent the $\operatorname{Pr}$ number for water. Figure 3 presents that the velocity of upper fluid is decreasing while velocity of lower fluid 


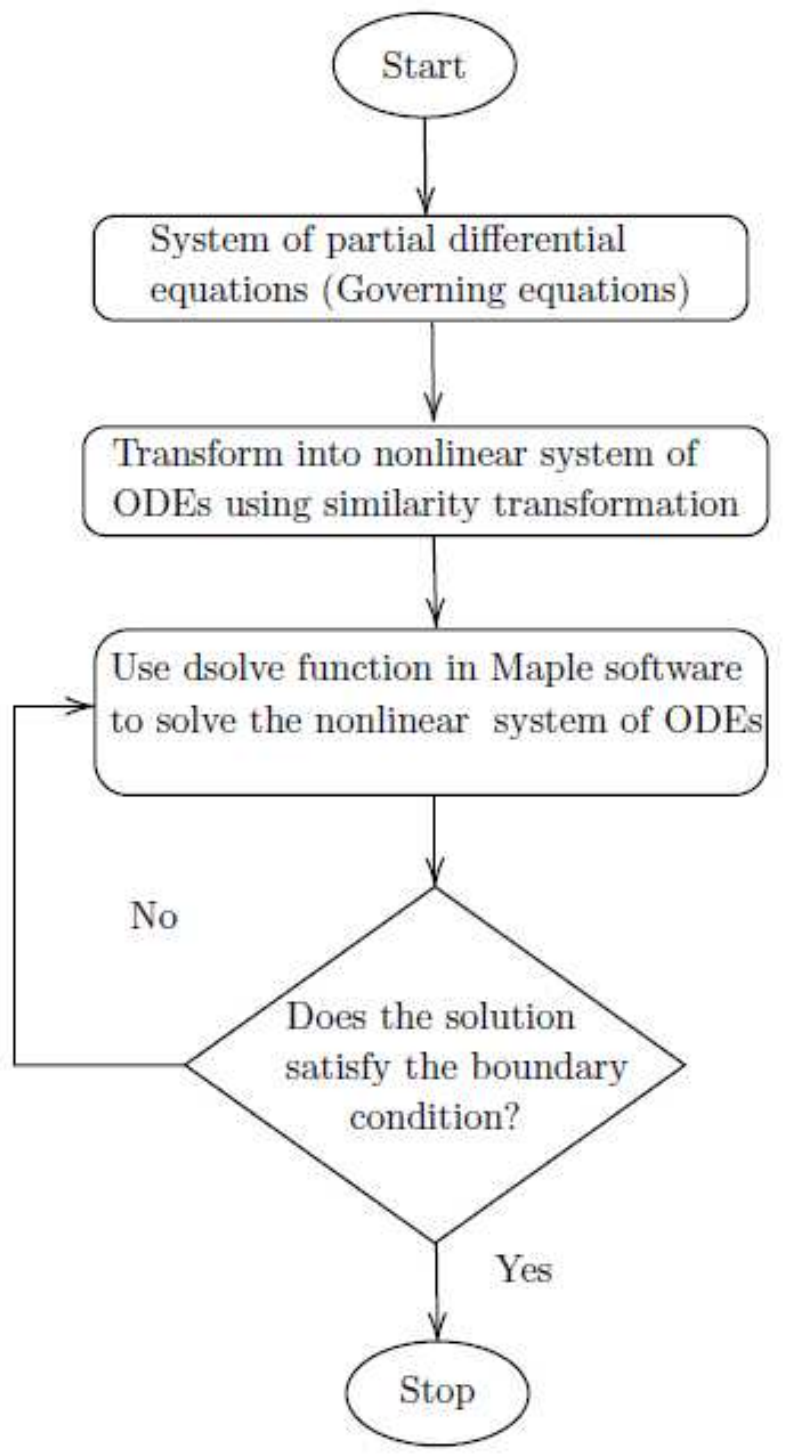

Figure 2: Implemented Algorithm to Solve the Mathematical Model

is increasing as micropolar parameter $K$ increases. We also notice that the boundary layer thickness is greater in lower fluid than in upper fluid. Besides, the values of skin friction of upper fluid is decreasing despite the skin friction of lower fluid increasing when micropolar parameter $K$ increases as in Table 2 . Referring to figure 4, we observe the trend of microrotation of upper fluid is decreasing whereas the values of microrotation of lower fluid is increasing as micropolar parameter $K$ increases. Both figures have a profile that achieving the maximum value nearby the wall which later declining to zero as boundary layer increase. Figure 5 unveils that the increment of micropolar parameter $K$ is enhancing the magnitude of temperature for upper fluid. It is observed that the temperature of lower fluid is decreasing due to the addition of micropolar parameter $K$ as shown in the same figure. 
Table 1: Values of $f^{\prime \prime}(0)$ for Various Values of $\lambda$ when $n=0, \operatorname{Pr}=1$

\begin{tabular}{|c|l|l|}
\hline$\lambda$ & $\begin{array}{l}\text { Rosali et al. } \\
{[36]}\end{array}$ & Present \\
\hline 0.0 & 1.232588 & 1.232590 \\
\hline 0.1 & 1.146561 & 1.146560 \\
\hline 0.2 & 1.051130 & 1.051130 \\
\hline 0.3 & 0.946816 & 0.946817 \\
\hline 0.4 & 0.834072 & 0.834073 \\
\hline 0.5 & 0.713295 & 0.713295 \\
\hline 1.0 & 0 & 0 \\
\hline 2.0 & -1.887307 & -1.887310 \\
\hline 3.0 & -4.276541 & -4.276540 \\
\hline 4.0 & -7.086378 & -7.086378 \\
\hline 5.0 & -10.264749 & -10.264700 \\
\hline
\end{tabular}

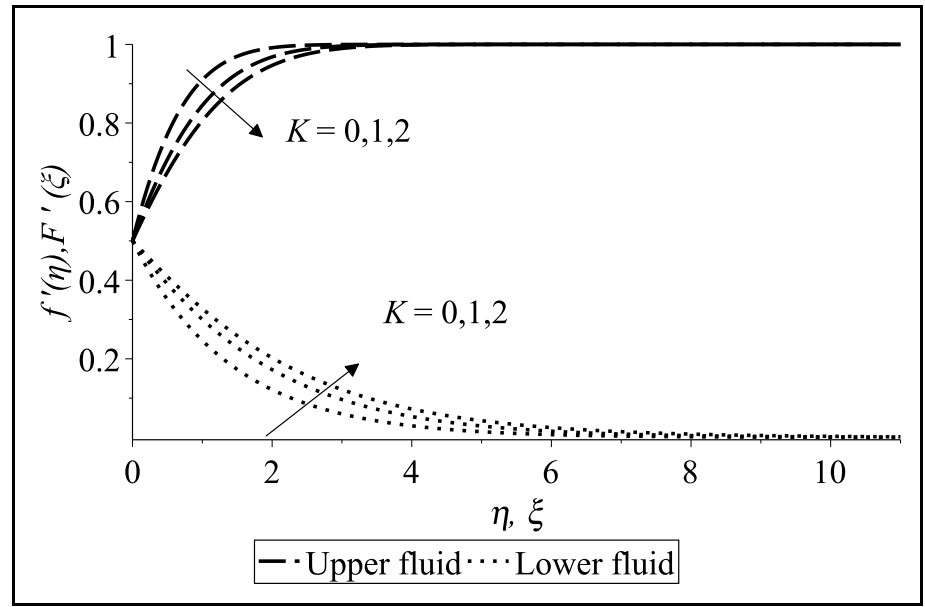

Figure 3: Velocity Profiles for Upper Fluid and Lower Fluid with Different Values of $K$ when $n=0, \lambda=0.5$ and $\operatorname{Pr}=7$

Table 2: Skin Friction with Different Values of $K$ when $n=0, \lambda=0.5, \operatorname{Pr}=7$

\begin{tabular}{|c|c|c|}
\hline$K$ & Upper fluid & Lower fluid \\
\hline 0.0 & 0.713295 & -0.353556 \\
\hline 1.0 & 0.490518 & -0.238570 \\
\hline 2.0 & 0.387729 & -0.187166 \\
\hline
\end{tabular}

In figures 6 - 8, the velocity, microrotation and temperature of a micropolar fluid of another quiescent fluid are presented for various Pr values when micropolar parameter $K=1$ with concentration $n=0$ (strong concentration) and stretching parameter $\lambda=0.5$. There is no change in velocity as in figure 6 for upper fluid and lower fluid when Pr number increases due to the decouple of boundary layer governing equation (2) - (4). Similar trend also appears for microrotation of upper fluid and lower fluid as in figure 7. Furthermore, figure 8 exhibits that the 


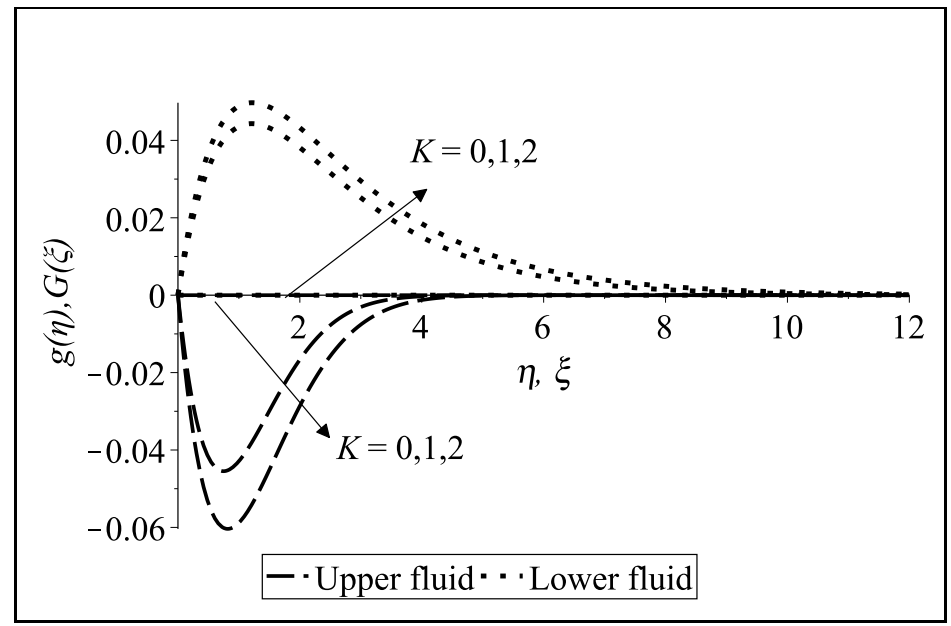

Figure 4: Microrotation Profiles for Upper Fluid and Lower Fluid with Different Values of $K$ when $n=0, \lambda=0.5$ and $\operatorname{Pr}=7$

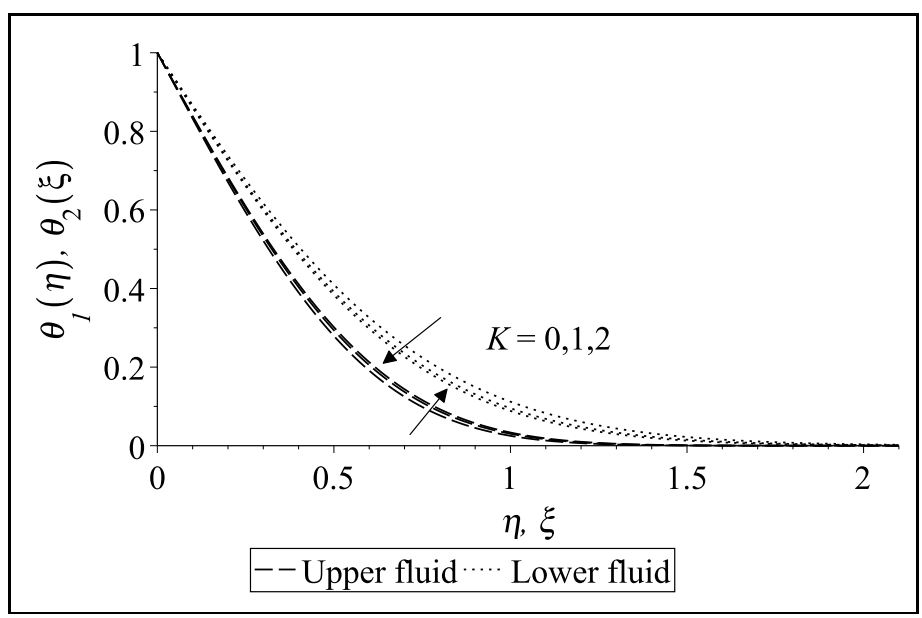

Figure 5: Temperature Profiles for Upper Fluid and Lower Fluid with Different Values of $K$ when $n=0, \lambda=0.5$ and $\operatorname{Pr}=7$ 
temperature of upper fluid and lower fluid is decreasing as Pr number gets higher. The higher Pr number, the fluid become more viscous resulting less heat lead to the lower temperature. It is also seen that lower fluid has more thermal boundary layer thickness compared to upper fluid.

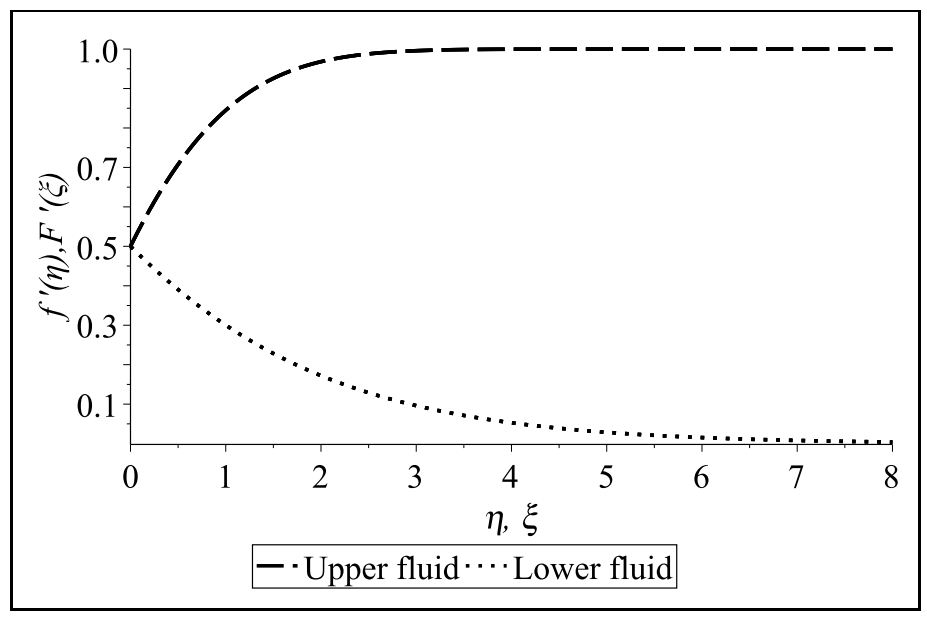

Figure 6: Velocity Profiles for Upper Fluid and Lower Fluid with $\operatorname{Pr}=0.5,1.0,2.0$ when $n=0$, $\lambda=0.5$ and $K=1$

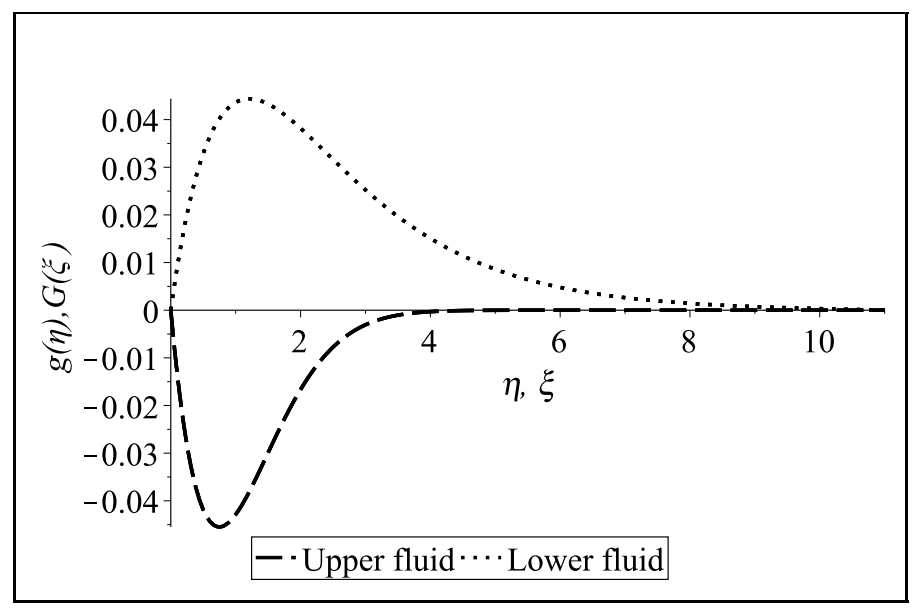

Figure 7: Microrotation Profiles for Upper Fluid and Lower Fluid with $\operatorname{Pr}=0.5,1.0,2.0$ when $K=1, n=0, \lambda=0.5$ 


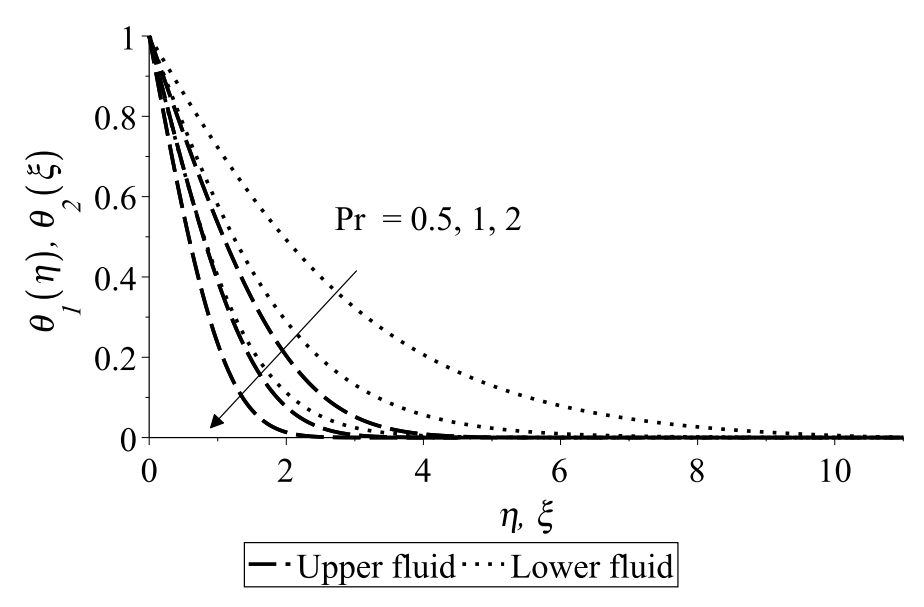

Figure 8: Temperature Profiles for Upper Fluid and Lower Fluid with $\operatorname{Pr}=0.5,1.0,2.0$ when $K=1, n=0, \lambda=0.5$

The graphical representation for velocity, microrotation and temperature when micropolar parameter $K=1$ with concentration $n=0.5$ (weak concentration), stretching parameter $\lambda=0.5$ and variation $\operatorname{Pr}$ number are depicted in figures 9 - 11. In figure 9 , we observe the changes in Pr values does not affect velocity for upper fluid and lower fluid as mentioned before in the case of $n=0$ (figure 6). Besides, the microrotation of upper fluid and lower fluid as show in figure 10 also follow the same trend. However, it is seen that the values of microrotation of upper fluid is increasing when concentration $n=0.5$ unlike when $n=0$ (figure 7 ). On the other hand, magnitude of microrotation for lower fluid is gradually decline toward zero. Figure 11 is plotted to present the temperature of upper fluid and lower fluid. It is seen that the temperature of upper fluid and lower fluid is decreasing along the addition of Pr number.

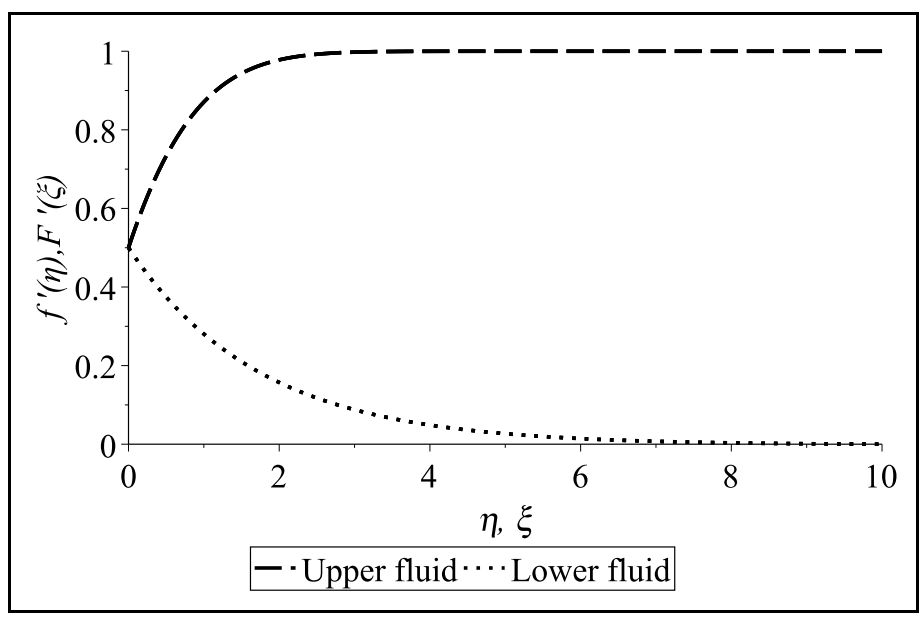

Figure 9: Velocity Profiles for Upper Fluid and Lower Fluid with $\operatorname{Pr}=0.5,1.0,2.0$ when $n=0.5$, $\lambda=0.5$ and $K=1$ 


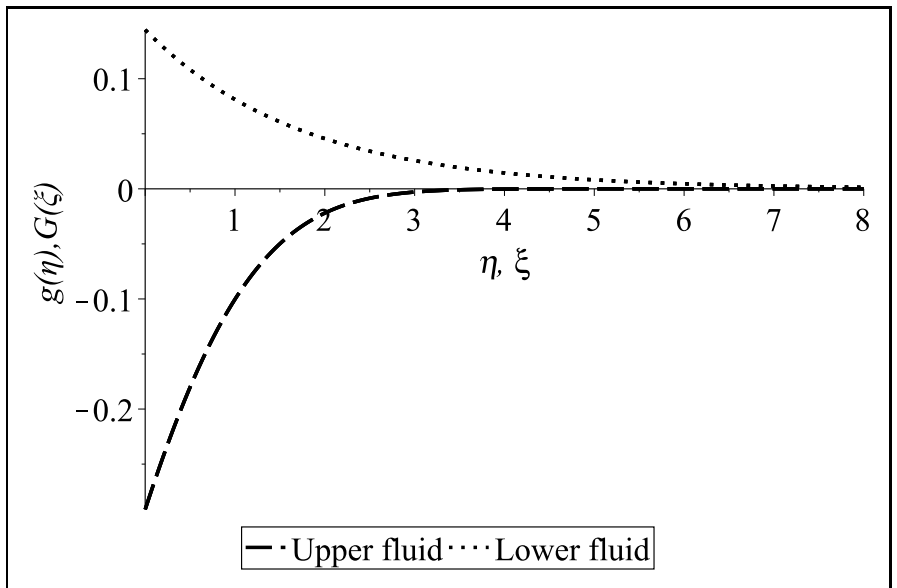

Figure 10: Microrotation Profiles for Upper Fluid and Lower Fluid with $\operatorname{Pr}=0.5,1.0,2.0$ when $n=0.5, \lambda=0.5$ and $K=1$

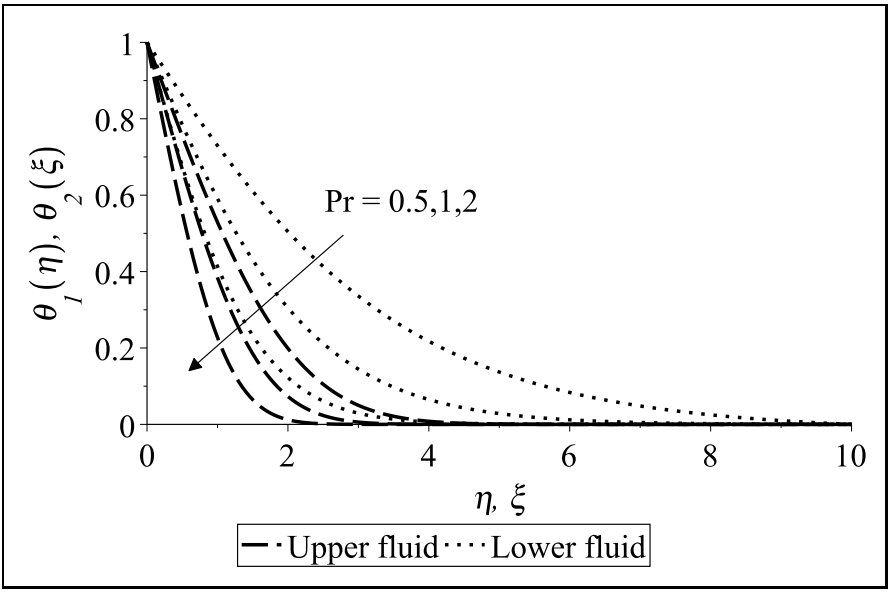

Figure 11: Temperature Profiles for Upper Fluid and Lower Fluid with $\operatorname{Pr}=0.5,1.0,2.0$ when $n=0.5, \lambda=0.5$ and $K=1$

Figures 12 - 17 represent the velocity, microrotation and temperature of upper fluid and lower fluid when micropolar parameter $K=1$ with concentration $n=0, \operatorname{Pr}=7$ when stretching parameter $\lambda$ varies. The velocity of upper fluid significantly increases when stretching parameter $\lambda$ increases as plotted in figure 12. From the same figure, the velocity of upper fluid is constant at stretching parameter $\lambda=1$ due to the equilibrium of velocity of stretching surface and the velocity of upper fluid. It is also shown in figure 13, the values of velocity for lower fluid raises with the increment of stretching parameter $\lambda$. We found that the forced is exerted by stretching surface enhanced the velocity of fluid. Generally, microrotation of upper fluid increases with the increment of stretching parameter and displayed parabolic profile as in figure 14. However, microrotation had positive values when stretching parameter $\lambda>1$ and negative value when stretching parameter $\lambda<1$. Figure 15 provides that the microrotation of lower fluid also increases as stretching parameter $\lambda$ increases. It is observed that microrotation of lower fluid 
exhibits positive values only which is contrary with upper fluid. From the same figure, we can see that the microrotation converge faster when stretching parameter $\lambda=4$ and 5 . In figure 16, there is a clear trend of decreasing values of temperature when stretching parameter $\lambda$ increases. The temperature of lower fluid also behaves in the similar manner as upper fluid with the increment of stretching parameter $\lambda$ but with greater thermal boundary layer thickness as plotted in figure 17. Based on the simulation that we performed, it is nearly impossible to solve the system of governing equation (2) - (4) using this method when there is no stretching activity (strecthing parameter $\lambda=0$ ).

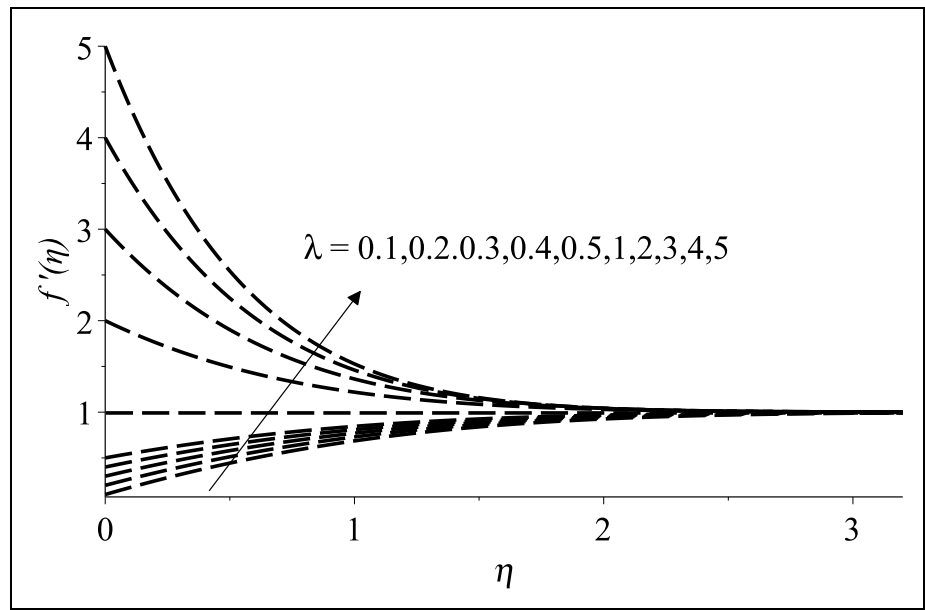

Figure 12: Velocity Profiles for Upper Fluid with Different Values of $\lambda$ when $K=1, n=0$, $\operatorname{Pr}=7$

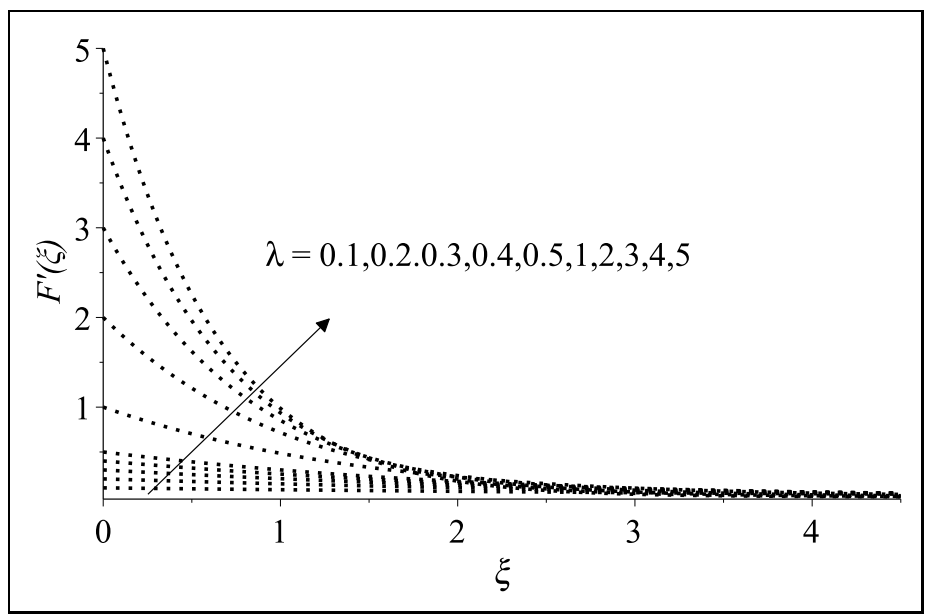

Figure 13: Velocity Profiles for Lower Fluid with Different values of $\lambda$ when $K=1, n=0$, $\mathrm{Pr}=7$ 


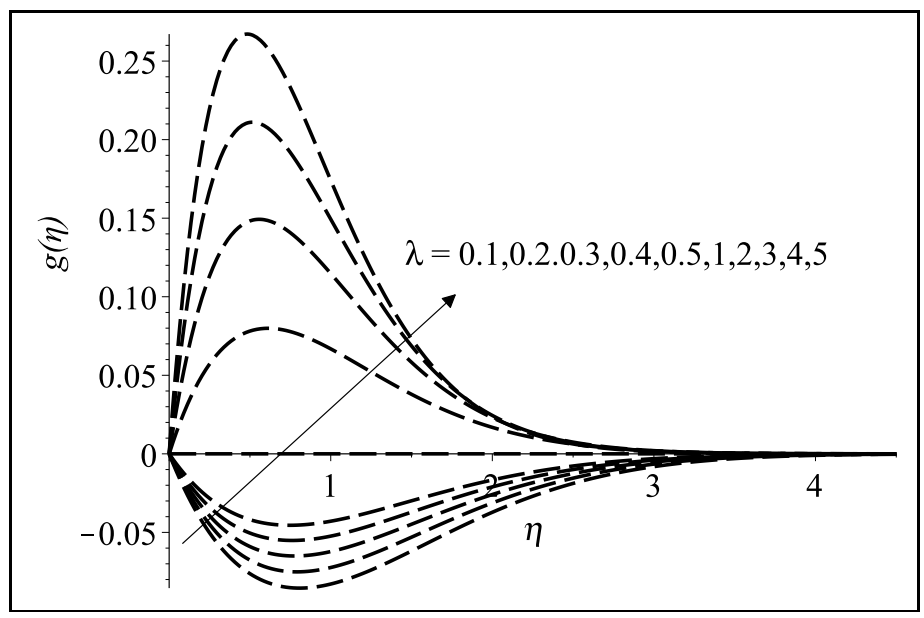

Figure 14: Microrotation Profiles for Upper Fluid with Different Values of $\lambda$ when $K=1$, $n=0, \operatorname{Pr}=7$

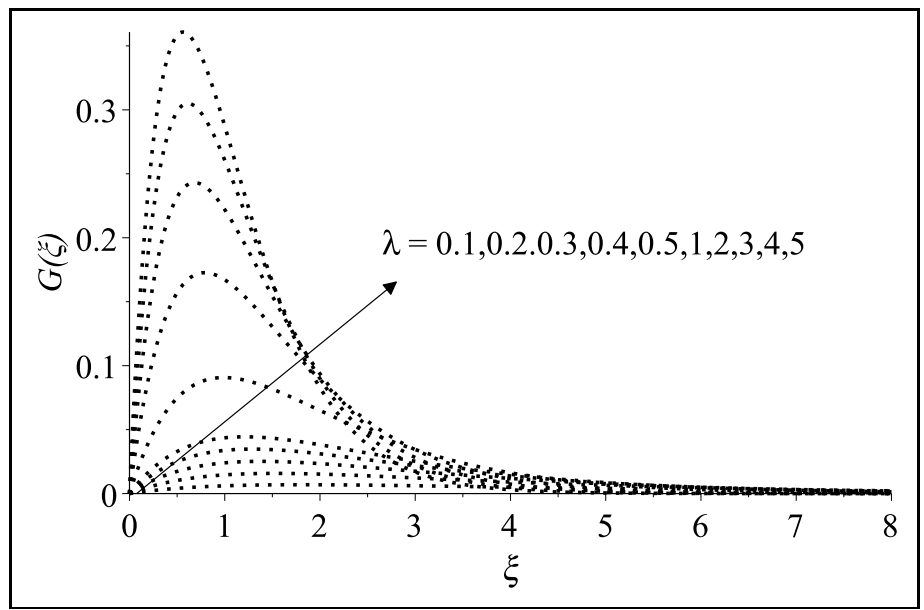

Figure 15: Microrotation Profiles for Lower Fluid with Different Values of $\lambda$ when $K=1$, $n=0, \operatorname{Pr}=7$ 


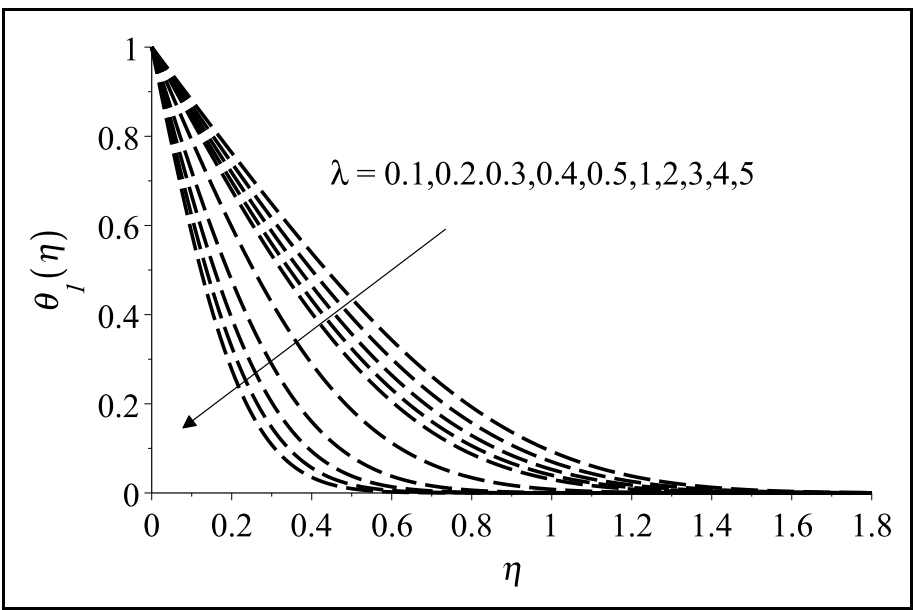

Figure 16: Temperature Profiles for Upper Fluid with Different Values of $\lambda$ when $K=1, n=0$, $\operatorname{Pr}=7$

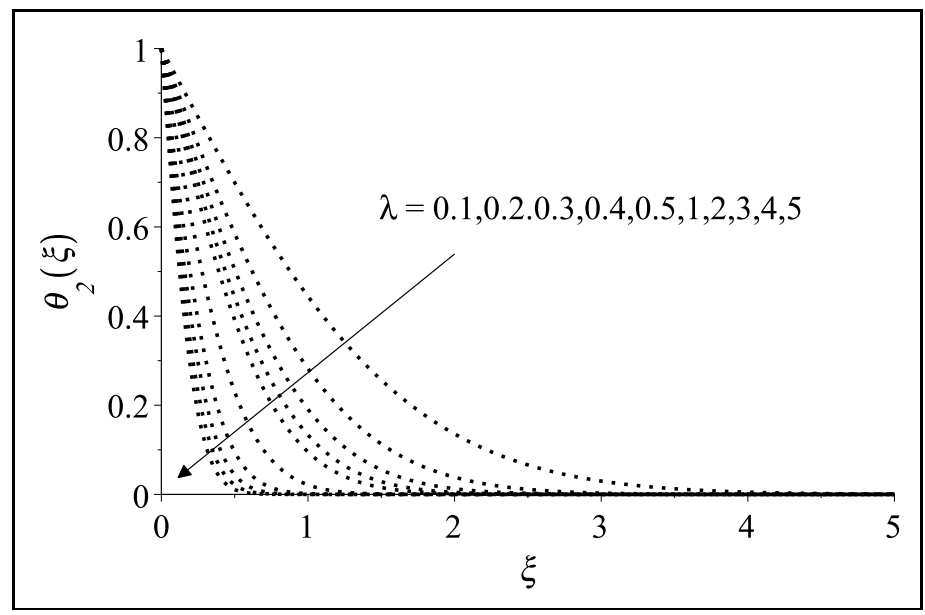

Figure 17: Temperature Profiles for Lower Fluid with Different Values of $\lambda$ when $K=1, n=0$, $\operatorname{Pr}=7$

\section{Conclusion}

In this paper, we have solved the problem of forced convection of micropolar fluid over stretching surface of another quiescent fluid. The governing boundary layer equations have been solved using dsolve function in Maple software version 2016. The numerical results acquired were velocity, microrotation and temperature for micropolar fluid with another quiescent fluid. With presence of forced convection, the velocity and microrotation of upper fluid decrease 
as micropolar parameter $K$ increases with stretching parameter $\lambda=0.5$. Besides, temperature of upper fluid slightly increases with increasing of micropolar parameter $K$. However, lower fluid reacts the opposite way under the same condition. As the stretching parameter increases, the velocity and microrotation of upper fluid and lower fluid increase due to the force exerted by stretching surface. Meanwhile the temperature of micropolar fluid decreases gradually. We also found that lower fluid has greater boundary layer thickness and thermal boundary layer thickness compared to upper fluid.

\section{Acknowledgement}

The authors would like to acknowledge the Research Management Centre-IIUM (C-RIG18004-0004), Ministry of Education (FRGS19-190-0799) and Research Management Centre-UTM (vote numbers 07G70, 07G72, 07G76 and 07G77) for the financial support in this research.

\section{References}

[1] Rees, D. A. S. \& Bassom, A. P. The Blasius boundary layer flow of a micropolar fluid. International Journal of Engineering. 1996. 34(1): 113-124.

[2] Ahmadi, G. Self-similar solution of incompressible micropolar boundary layer flow over a semi-infinite plate. International Journal of Engineering Science. 1976. 14(7): 639-646.

[3] Chawla, S. S. Boundary layer growth of a micropolar fluid. International Journal of Engineering Science. 1972. 10(11): 981-987.

[4] Guram, G. S. \& Smith, A. C. Stagnation flows of micropolar fluids with strong and weak interactions. Computers and Mathematics with Applications. 1983. 6(2) :213-233.

[5] Peddieson, J. An application of the micropolar fluid model to the calculation of a turbulent shear flow. International Journal Engineering Science. 1972. 10(1): 23-32.

[6] Takhar, H. S. \& Soundalgekar, V. Boundary Layer Flow of A Micropolar Fluid - Non Similar Solution. Mechanics Research Communications. 1986. 13(5): 255-263.

[7] Ishak, A., Nazar, R. \& Pop, I. Boundary-layer flow of a micropolar fluid on a continuously moving or fixed permeable surface. International Journal of Heat and Mass Transfer. 2007. 50(2324): 4743-4748.

[8] Ariffin, N. M., Arifin, N. M. \& Bachok, N. Marangoni boundary layer flow in micropolar fluid with suction or injection. AIP Conference Proceedings. 2017. 1795: 020011.

[9] Shelukhin, V. V. \& Neverov V. V. Flow of micropolar and viscoplastic fluids in a Hele-Shaw cell. Journal of Applied Mechanics and Technical Physics. 2014. 55(6): 905-916.

[10] Ariman, T., Turk, M. A. \& Sylvester, N. D. Microcontinuum fluid mechanics- a review. International Journal Engineering Science. 1973. 11: 905-930.

[11] Ariman, T., Turk, M. A. \& Sylvester, N. D. Applications of microcontinuum fluid mechanics. International Journal Engineering of Science. 1974. 12(6): 273-293.

[12] Willson, A. J. Boundary layers in micropolar liquids. Mathematical Proceedings of the Cambridge Philosophical Society. 1970. 67(2): 469. 
[13] Lukaszewicz, G. Micropolar Fluids Theory and Applications. (N. Bellomo \& P. Torino, Eds.). Birkhauser. 1999.

[14] Eremeyev, V. A., Lebedev, L. P. \& Altenbach, H. Foundations of Micropolar Mechanics. Springer Berlin Heidelberg. 2013.

[15] Attia, H. A. Stagnation point flow and heat transfer of a micropolar fluid with uniform suction or blowing. Journal of the Brazilian Society of Mechanical Sciences and Engineering. 2008. 30(1): 57-65.

[16] Eringen, A. C. Theory of thermomicrofluids. Journal of Mathematical Analysis and Applications. 1972. 38(2): 480-496.

[17] Gorla, R. R. S. R., Pender, R. \& Eppichs, J. Heat transfer in micropolar boundary layer flow over a flat plate. International Journal Engineering of Science. 1983. 21(7): 791-798.

[18] Balaram, M. \& Sastri, V. U. K. Micropolar free convection flow. International Journal of Engineering Sciences. 1973. 16: 437-441.

[19] Cheng, C. Y. \& Wang, C. C. Forced convection in micropolar fluid flow over a wavy surface. Numerical Heat Transfer, Part A: Applications: An International Journal of Computation and Methodology. 2010. 37(3): 271-287.

[20] Sakiadis, B. C. Boundary layer behavior on continuous solid surfaces. American Institute of Chemical Engineering Journal. 1961. 7(1): 26-28.

[21] Crane, L. J. Flow past a stretching plate. Zeitschrift Fr Angewandte Mathematik Und Physik ZAMP. 1970. 21(4): 645-647.

[22] Miklavcic, M. \& Wang, C. Y. Viscous flow due to a shrinking sheet. Quarterly of Applied Mathematics. 2006. 64(2): 283-290.

[23] Soundalgekar, V. \& Takhar, H. S. Flow of micropolar fluid past a continuously moving plate. International Journal Engineering Science. 1983. 21(8): 961-965.

[24] Chiam, T. C. Micropolar fluid flow over a stretching sheet. Journal of Applied Mathematics and Mechanics. 1982. 62: 565-568.

[25] Na, T., \& Pop, I. Boundary-layer flow of a micropolar fluid due to a stretching wall. Archive of Applied Mechanics. 1997. 67: 229-236.

[26] Ishak, A., Lok, Y. Y. \& Pop, I. Stagnation-point flow over a shrinking sheet in a micropolar fluid, Chemical Engineering Communications. 2010. 197(11): 1417-1427

[27] Yacob, N. A. \& Ishak, A. Micropolar fluid flow over a shrinking sheet. Meccanica. 2012. 47(2): 293-299.

[28] Wang, C. Y. Stagnation flow on the surface of a quiescent fluid - an exact solution of the Navier-Stokes Equation. Acta Technica . 1988. 33(2): 715-730.

[29] Reza, M. \& Gupta, A. S. MHD stagnation-point flow of an electrically conducting fluid on the surface of another quiescent fluid. Acta Mechanica. 2012. 223(11): 2303-2310.

[30] Rohni, A. M., Omar, Z. \& Man, N. H. Dual solutions of stagnation-point flow of a fluid on a shrinking surface of another quiescent fluid. In 4th Intl Conference on Advances in Engineering Sciences \&6 Applied Mathematics (ICAESAM2015). 2015. 2: 74-76. 
[31] Reza, M., Panigrahi, S. \& Mishra, A. K. Stagnation point flow and heat transfer for a viscoelastic fluid impinging on a quiescent fluid. Sadhana - Academy Proceedings in Engineering Sciences. 2017. 42(11): 1979-1986.

[32] Isa, S. M. \& Mohammad, N. F. Boundary layer flow of dusty on a stretching sheet of another quiescent fluid. Journal of Physics: Conference Series. 2017. 819(1).

[33] Jena, S. K. \& Mathur, M. N. Similarity solutions for laminar free convection flow of a thermomicropolar fluid past a non-isothermal vertical flat plate. International Journal of Engineering Science. 1981. 19(11): 1431-1439.

[34] Tsou F. K., Sparrow E. M. \& Goldstein R. J. Flow and heat transfer in the boundary layer on a continuous moving surface. Int. J. Heat Mass Transfer. 1967. 10: 219-35.

[35] Lok, Y., Amin, N. \& Pop, I. Unsteady boundary layer flow of a micropolar fluid near a stagnation point with uniform suction or injection. Jurnal Teknologi. 2007. 46: 15-32.

[36] Rosali, H., Ishak A. \& Pop, I. Micropolar fluid flow toward a strecthing or shrinking sheet in a porous medium with suction. International Journal of Engineering Science. 2012. 21(8): 961-965.

[37] Turyilmazoglu, M. Flow of a micropolar fluid due to a porous stretching sheet and heat transfer. International Journal of Non-Linear Mechanics. 2016. 83: 59-64. 